\author{
TRIBUNAL DE JUSTIÇA DO ESTADO DA PARAÍBA \\ Acórdão do processo nº 01720110000100001 \\ Órgão: Quarta Câmara Cível \\ Relator: Frederico Martinho da Nóbrega Coutinho \\ Julgamento: 03/04/2012
}

MEIOS DE CONTROLE EM DIREITO SANITÁRIO. VIGILÂNCIA SANITÁRIA. LICENÇA DE FUNCIONAMENTO. DISTÂNCIA MÍNIMA. ART. 79, DA LEI ESTADUAL N 7.668/04. INCONSTITUCIONALIDADE INCIDENTAL. PRINCÍPIO DA LIVRE CONCORRÊNCIA. ENTENDIMENTO SUMULADO PELO SUPREMO TRIBUNAL FEDERAL. PRECEDENTES DESTA CORTE DE JUSTIÇA. - O limite geográfico resguardando-se a distância mínima de 500 (quinhentos) metros, contados a partir do estabelecimento com registro mais antigo no órgão de controle Sanitário Estadual viola e atinge o exercício da livre concorrência, sendo uma manifestação do princípio constitucional da liberdade de iniciativa econômica privada. - Mantém-se a decisão que concedeu a segurança, quando há a comprovação do direito líquido e certo da parte impetrante, negando-se provimento à Remessa Oficial.

\title{
COMENTÁRIO
}

\section{RISCOS DO CONTROLE E CONTROLE DOS RISCOS}

\author{
Risk of control and control of the risk
}

\author{
Nicolina Silvana Romano-Lieber* \\ Renato Rocha Lieber**
}

\footnotetext{
Livre Docente; Professora Associada, Departamento de Prática de Saúde Pública, Faculdade de Saúde Pública, Universidade de São Paulo. Farmacêutica Bioquímica. São Paulo/SP - Brasil. E-mail: nicolina@usp.br

** Doutor em Saúde Pública; Professor Doutor, Departamento de Produção, Faculdade de Engenharia de Guaratinguetá, Universidade Estadual Paulista. Engenheiro Químico e Ambiental. Guaratinguetá/SP - Brasil.
}

Artigo recebido em: 08/10/2013. Aprovado em: 12/11/2013. 


\section{RESUMO}

A constatação da ilegalidade das restrições geográficas para farmácias oferece oportunidade para se entender melhor a natureza desse estabelecimento comercial, integrado no sistema de saúde. A revisão histórica mostra que os perigos associados ao preparo e a disponibilização de medicamentos passaram a ser controlados por regulamentação do Estado a partir do século XIII na Europa. No sistema de guilda, restrições foram impostas, não só para o exercício profissional, mas também para preços e localização geográfica. Seu propósito foi excluir incertezas na saúde pública através da garantia da qualidade e da possibilidade de acesso ao medicamento. Todavia, essas condições mudaram ao longo do século $\mathrm{XX}$, com a prevalência de medicamentos industrializados à disposição via economia de mercado. Nesta, os melhores termos de troca se dão preferencialmente sob aglomeração geográfica, que reduz as expectativas de risco dos envolvidos, baixando os preços. De maneira geral, não há consenso internacional em se continuar mantendo ou não as restrições geográficas. Mas o custo crescente da universalização do acesso à saúde vem tornando necessária a revisão da segurança proporcionada pelas restrições profissionais em prol de uma lógica de incerteza configurada em risco.

Palavras-chave: Atenção Farmacêutica; Farmácia; Incerteza Jurídica; Livre concorrência; Zoneamento.

\section{ABSTRACT}

The decision of the Paraíba State's Court to not obstruct the geographical concentration of pharmacies offers opportunity to better understand the nature of this commercial establishment, integrated to the health system. The historical review shows that the hazards associated with the preparation and availability of drugs began to be controlled by state regulations in the $13^{\text {th }}$ century in Europe. In the guild system, restrictions were imposed, not only for practice, but also for pricing and geographic location. Its purpose was to exclude uncertainties in public health by ensuring the quality and the possibility of access to medicines. However, these conditions have changed through the $20^{\text {th }}$ century, with the prevalence of manufactured drugs available via market economy. In it, the best terms of trade are given preferably within cluster, which reduces the risk expectations of those involved, lowering prices. In general, there is no international consensus on whether or not to maintain geographical restrictions. Nevertheless, the rising cost of universal access to health care makes it necessary to review the security provided by professional restrictions on behalf of a logic of uncertainty configured at risk.

Keywords:.Cluster; Pharmaceutical Care; Pharmacy; Free Market; Juridical Uncertainty.

\section{Introdução}

Adoção de medidas de controle de natureza sanitária, como a restrição geográfica para farmácias, envolve conflitos. Estes, com frequência, quando levados a juízo, ofuscam questões essenciais subjacentes que, no contexto da demanda, 
se mostram aparentemente de pouca pertinência. Todavia, pode-se melhor entender uma decisão desse tipo, ou suas repercussões, contextualizando-se os acertos propostos em termos de seus significados, pressupostos e trajetos históricos. Pretende-se mostrar como, no problema em questão, o pressuposto da adoção ou rejeição de uma norma para controle pode pôr em risco aquilo mesmo que se pretendia controlar, no caso, as melhores condições de acesso a medicamentos e serviços farmacêuticos de qualidade pela população.

\section{Risco como pressuposto esquecido}

Adoção ou rejeição de medidas de controle envolve incertezas raramente explícitas. Propostas costumam ser formuladas com base na experiência, na história, na cultura expressa no senso comum e, mais raramente, com base em procedimentos científicos. Nestes, os pressupostos de causa e efeito configuram-se quase sempre em condições muito restritas, impostas e, na maior parte das vezes, em relação muito difícil com as condições contingentes do mundo. Quando a incerteza decorrente é formulada num contexto de prognósticos, configura-se a situação de risco. (1) Não sem razão, medidas sanitárias voltadas à preservação da saúde pública são mais bem estabelecidas sob a lógica de risco e, no nosso país, em particular, isso se traduz em lei.(2) Todavia, para a autoridade sanitária, assim como para qualquer outra em nossa particularidade, há desconforto no contingenciamento do controle. Normas, regulamentos e restrições acabam se impondo indiferentemente às circunstâncias. Com isso, furta-se ao arbítrio necessário e obliteram-se as incertezas inerentes associadas à condição de risco. Não é sem razão, portanto, a taxa de demandas judiciais em nosso país.

No sistema legal, pressupõe-se que o risco possa ser controlado sob duas condições: ou por responsabilidade civil para compensação das vítimas ("constrangimento de mercado") ou por regulação ("constrangimento específico").(3) A sentença, objeto deste comentário, insere-se nesse último caso. Ela traz à tona, não apenas, as dificuldades para se entender o que são hoje as farmácias no Brasil: estabelecimentos comerciais ou estabelecimentos de prestação de serviços de saúde, ou mesmo ambos. Mas também, evidencia, em sua decisão, que esse (des)entendimento não pode ser desconsiderado, sob o risco de a ação de controle do Estado infringir o interesse coletivo maior, previsto na Carta Magna.

\footnotetext{
1 A formulação científica de prognósticos decorre do uso de teorias científicas. O seu caráter provisório é a condição necessária para a possibilidade do desastre envolvendo uso de tecnologia. (LIEBER R.R.; ROMANO-LIEBER N.S. Risco e precaução no desastre tecnológico. Cadernos Saúde Coletiva, Rio de Janeiro, v.13, n.1, p.67-84, 2005).

2 BRASIL. Lei $n^{\circ} 8.080$, de 19 de setembro de 1990. "Dispõe sobre as condições para a promoção, proteção e recuperação da saúde, a organização e o funcionamento dos serviços correspondentes e dá outras providências.". Disponível em: <http://www.planalto.gov.br/ccivil_03/leis/l8080.htm>. Acesso em: 19 jan. 2014.

${ }^{3}$ CALABRESE apud ABEL, R. L. A socialist approach to risk. Maryland Law Review, Maryland, v.41, p. $697,1981-1982$.
} 
A improcedência legal de se impor disposição geográfica desses estabelecimentos é, pela sentença, coisa julgada. Todavia, há ainda uma outra perspectiva a se considerar. Como a condição de risco é parte essencial da lógica sanitária, quais seriam as inferências possíveis sob esse ponto de vista?

Demandas no setor saúde caracterizam necessidades que a lei ou a norma em si frequentemente são insuficientes para atender o que é exigido. A excepcionalidade de se estar doente aloca reflexões de natureza cultural, ética, política ou da constituição social entre muitas outras, sem, é claro, excluir as questões técnicas e econômicas. Esses aspectos estão em constante transformação no âmbito social, muitas vezes em descompasso entre si. Não cabe aqui considerar cada um deles em detalhe. Todavia, uma breve revisão histórica da atividade farmacêutica, como comércio e profissão, pode ajudar a se entender melhor de que forma essas transformações acabam resultando em conflitos, muitas vezes sujeitos à mediação pelo direito.

\section{História da farmácia ou uma história de controle de risco}

A noção de cura por meio da administração de um agente físico, externo ao corpo, remonta à pré-história. Religião e magia fizeram parte dessa história durante a antiguidade até a idade média, quando pode ser registrado o advento de figuras profissionais diferenciadas como o médico, o herborista, o droguista e o boticário. ${ }^{(4)}$ Em princípio, todas elas se prestavam ao mesmo propósito indistintamente, diagnosticando, prescrevendo e preparando produtos destinados à cura. Os médicos, em destaque, gozavam de um prestígio maior, por sua atividade predominantemente intelectual em contraste com os demais, os comerciantes, os preparadores (boticários) ou os ligados ao manuseio impuro, como no caso dos cirurgiões barbeiros. ${ }^{(5)}$

As guildas, ou corporações de ofício desses profissionais, buscavam, junto às cidades, a regulamentação do exercício do ofício, de forma a excluir a concorrência por outros praticantes e, principalmente, garantir os privilégios de uso da mão de obra aprendiz. ${ }^{(6)} \mathrm{O}$ arranjo em proximidade espacial, como aqui se discute, foi sempre prática comum, como forma de melhor alcançar esses objetivos. ${ }^{(7)}$

\footnotetext{
${ }^{4}$ A função "boticário", em particular, tem origem nas contribuições árabes ao ocidente. Os mesmos mantinham uma atividade comercial regular associada a preparação de fórmulas. Na idade média essa prática combinou-se com as antigas compilações gregas (Matéria Médica), trazendo alguma distinção na prática de médicos e boticários. (COWEN, D. L.; HELFAND, W.H. Pharmacy: an illustrated history. New York: Abrams Inc; 1990. p. 57).

${ }_{5}$ SCHEPERS, R. Pharmacists and medical doctors in nineteenth century Belgium. Sociology of Health \& IIIness, Leeds, v. 10, n. 1, p. 68-90, 1988.

${ }^{6}$ EPSTEIN, S.R. Craft guilds, apprenticeship, and technological change in preindustrial Europe. Journal of economic history, Cambridge, v. 58, p. 684-713, 1998;

7 A preferência no arranjo convinha tanto ao artesão quanto ao consumidor, ao permitir principalmente a redução de assimetrias nas informações, relativas às técnicas, preços e padrões de qualidade. EPSTEIN. S. R. op.cit.
} 
O mesmo deriva de antigas tradições árabes de conglomerado, como o bazar, prestando-se também à melhor logística no suprimento de matéria-prima. ${ }^{(8)}$

No ocidente, o marco regulatório foi o decreto, em 1240, de Frederico II, imperador do Sacro Império Romano-germânico. Nele, separaram-se claramente as atribuições do médico e do farmacêutico. Estabeleceram-se proibições. $O$ médico ficou desautorizado a dispensar produtos, enquanto o farmacêutico ficou proibido de diagnosticar doenças e prescrever os remédios. O decreto impôs aos farmacêuticos o tabelamento de preços, inspeções médicas, restrições geográficas em função da densidade populacional e licenças de funcionamento. Em contrapartida ao privilégio, os farmacêuticos deveriam prestar juramento e manter a qualidade e a segurança dos produtos manuseados. ${ }^{(9)}$

O decreto de Frederico II profissionalizou o farmacêutico na estrutura da saúde pública e impôs o controle da atividade pelo Estado com o propósito de reduzir riscos e de garantir o amplo acesso. Essa lógica tornou-se referência para a Europa continental, sem alcançar, todavia, os países anglo-saxões. ${ }^{(10)}$ Durante a revolução francesa, mesmo com a abolição de todos os privilégios corporativos, esse em particular ainda foi mantido. ${ }^{(11)}$ A separação de atividades, todavia, foi flexível para atender os objetivos de acesso, permitindo muitas vezes a dispensação por médicos na condição de falta do farmacêutico. ${ }^{(12)}$ Na Alemanha, o papel regulador do Estado na farmácia só foi extinto com a ocupação americana após

\footnotetext{
${ }^{8}$ A conveniência de alocações em meio às principais rotas comerciais foi razão estratégica para assegurar suprimentos valiosos e freqüentemente raros, como nas farmácias no império otomano descrito por Düzbakar e Ercan. (DÜZBAKAR, Ö.; ERCAN, Ö. Notes on the Attar-Poets in ottoman history: reflections from the Shari'a court records of bursa and poets' biographies. Journal of the International Society for the History of Islamic Medicine, Istambul, v.5, p.19-24, 2006).

${ }^{9}$ COWEN, D. L.; HELFAND, W.H. op. cit., p. 55; COWEN, D. L. Pharmacists and physicians: an uneasy relationship. Pharmacy in History, Madison, v. 34, n. 1, p. 3-16, 1992; ANDERSON, S. The historical context of pharmacy. In: TAYLOR, K. MG; HARDING, G. (Ed.). Pharmacy Practice. London: Taylor \& Francis Press, 2001, p. 4. A atividade farmacêutica, ainda na prática do boticário, proporcionava por si mesma baixos rendimentos, obrigando a busca de outras fontes de renda para garantir a sobrevivência do membro da guilda. No século XIV, o monopólio dos boticários incluía a venda de livros e velas, por exemplo (COWEN, D. L.; HELFAND, W.H. op. cit., p. 53). No século XVIII, um catálogo de produtos no formato de "Materia Medica", distribuído nos EUA, lista os produtos farmacêuticos disponíveis, incluindo entre outros, perfumes, produtos químicos e artigos de mercearia (COWEN, D. L. A store mixt, various, universal. The Journal of the Rutgers University Libraries, New Brunswick, v. 25, n. 1, p. 1-9, Dec. 1961).

${ }^{10} \mathrm{Na}$ Inglaterra, Irlanda e Escócia prevaleceu a tradição liberal, de forma que foi garantido aos farmacêuticos o direito de prestarem consulta, diagnóstico e prescrição, embora restrita aos medicamentos dispensados. Em contrapartida, a comercialização de medicamentos ficou liberada a outros profissionais, incluindo médicos e químicos. Essa prática corrente foi importante até a instituição do sistema nacional de saúde que garantiu acesso médico a toda população Até o início do século XX, os médicos eram responsáveis pela maioria da dispensação, enquanto que os farmacêuticos atendiam principalmente os pobres. (ANDERSON, S. op. cit.).

${ }^{11}$ COWEN, D.L. Pharmacy and freedom. Pharmacy in History, Madison v. 26, n. 2, p. 70-82, 1984.

${ }^{12}$ Mesmo no século XVIII, na Bélgica, médicos e cirurgiões tinham o direito de dispensar nas localidades em que faltasse um boticário, direito que veio a ser abolido só a partir de 1953. (SCHEPERS, R. op.cit.).
} 
a Segunda Guerra Mundial, cujas leis liberaram toda a atividade de farmácia às condições de mercado. ${ }^{(13)}$

Nos países anglo-saxões a tradição liberal preferiu garantir o acesso pela ampla liberdade de comercialização de produtos farmacêuticos, possibilitando a configuração de redes ou cadeias comerciais. Até 1954, produtos farmacêuticos foram entendidos como produtos químicos e autorizados a serem vendidos quando supervisionados por um profissional não necessariamente farmacêutico. ${ }^{(14)}$

Transformações no século XX trouxeram novos desafios aos farmacêuticos, obrigando-os a repensar a natureza do seu papel social. Esse processo mostrou três fases distintas. ${ }^{(15)}$ A ocupação "boticário" converteu-se em "farmacêutico" na França do século XVIII. Caracterizado como profissional de saúde de formação superior, suas atribuições excluíam o diagnóstico e a prescrição. ${ }^{(16)}$ Todavia, a partir do início do século passado, o preparo de medicamentos pela manipulação vai dando lugar à comercialização de fármacos patenteados e produzidos em larga escala pelo processo industrial, implicando em gradativo esvaziamento das funções anteriores. ${ }^{(17)}$ No Brasil, isso ficou claro com a distinção entre farmácia e drogaria. ${ }^{(18)}$ Consequentemente, uma terceira fase se fez necessária, resgatando o relevo da atividade em termos de serviço de saúde, quando se propôs a farmácia clínica e a atenção farmacêutica.

\footnotetext{
${ }^{13}$ As leis para o setor farmacêutico foram objeto de sucessivos questionamentos, todos refutados pela suprema corte alemã. Em 1960, entretanto, a formação de redes foi proibida. (COWEN, D.L. Pharmacy and freedom, cit. e COWEN, D.L.; HELFAND, W.H. op.cit. p. 72).

${ }^{14}$ A prática ganhou relevo quando, a partir de 1870 , a loja de departamentos Harolds conquistou judicialmente o direito de comercializar medicamentos sob o título de "produtos químicos". A sobrevivência dos farmacêuticos tornou-se cada vez mais dependente de atividades outras no setor de serviços. No início do século XX a renda nos estabelecimentos farmacêuticos decorria principalmente de serviços odontológicos e fotográficos. Apenas $10 \%$ da renda vinha da dispensação de prescrições. (ANDERSON, S., op.cit.).

${ }^{15}$ HEPLER, C.D.; STRAND, L.M. Oportunidades y responsabilidades en la Atención Farmacéutica. American Journal of Hospital Pharmacy, Bethesda, v.47, p. 533-534, 1990.

${ }^{16}$ COWEN, D.L. Pharmacy and freedom, cit. França e Prússia dispunham de ensino superior regular para farmacêuticos desde o século XVII (COWEN, D. L.; HELFAND, W.H. op.cit.).

${ }_{17}$ Vieira relaciona a descaracterização da farmácia e do farmacêutico com a perda de prestígio social. Obviamente, tal perda, em prol dos ganhos de consumo promovidos pela industrialização, não foi exclusiva da profissão farmacêutica. Prestigio decorre da exposição ao risco em prol do benefício social. VIEIRA, F.S. Possibilidades de contribuição do farmacêutico para a promoção da saúde. Ciência \& Saúde Coletiva, Rio de Janeiro, v.12, n.1, p.213-220, 2007. No Reino Unido, farmacêuticos compartilham grande prestígio desde o século XVII, pois, ao contrário do médicos, mantiveram-se à serviço na cidade de Londres durante a grande peste negra de 1666. (COWEN, D. L.; HELFAND, W.H. op.cit., p. 72 e TOWNSEND, E. Future trends in general practice. The Journal of the College of General Practitioners, London, v. 5, n. 4, p. 501-24, 1962).

${ }^{18}$ BRASIL. Lei $n^{\circ} 5.991$, de 17 de dezembro de 1973. "Dispõe sobre o controle sanitário do comércio de drogas, medicamentos, insumos farmacêuticos e correlatos, e dá outras providências.". Disponível em: < http://www.planalto.gov.br/ccivil_03/leis/L5991.htm>. Acesso em: 19 jan. 2014.
} 


\section{Atenção farmacêutica e farmácia clínica}

A prática da farmácia clínica tem início na década de 1960. A rotina farmacêuti$\mathrm{ca}$, orientada para a atenção ao paciente e ao medicamento, passa a ser vista como um meio ou instrumento para se alcançar um resultado, seja este paliativo, curativo ou preventivo. Em outras palavras, a finalidade do trabalho farmacêutico deixa de pressupor foco no medicamento, como produto farmacêutico, e passa a ser direcionada ao paciente (lócus do medicamento), com a preocupação de que os riscos inerentes à utilização desse produto sejam minimizados. O serviço de atenção farmacêutica vai sendo definido e conceituado, inclusive no Brasil, enfatizando-se a ação do farmacêutico junto ao paciente para buscar o melhor resultado da farmacoterapia. ${ }^{(19)}$

O consenso brasileiro no assunto introduziu uma prática mais geral ao farmacêutico, pressupondo uma lógica de serviço como "cuidado farmacêutico", a ser integrado de forma sistêmica num conjunto mais amplo. Com isso, seguiu-se a tendência ideológica do sistema de saúde brasileiro (Sistema Único de Saúde [SUS]) e seus princípios doutrinários. ${ }^{(20)}$ Têm sido várias as iniciativas nesse sentido, (21) muito embora as farmácias continuem a ser vistas essencialmente como estabelecimentos comerciais. Pesquisas mostram que a atenção farmacêutica enfrenta dificuldades como falta de tempo para a atividade, falta de conhecimento dos farmacêuticos sobre o tema e falta de interesse dos usuários, ${ }^{(22)}$ além de falta de estrutura adequada para a prática. ${ }^{(23)}$

Nesses termos, poder-se-ia entender que a farmácia constitui um lócus de serviço que vai além da prática de comercialização. Embora toda prática comercial envolva alguma assistência, a assistência farmacêutica distingue-se por se

\footnotetext{
${ }^{19}$ Atenção farmacêutica vem sendo definida em diferentes instâncias. (HEPLER, C.D.; STRAND, L.M. op.cit.; ORGANIZACIÓN MUNDIAL DE LA SALUD. El papel del farmacéutico en el sistema de atención de salud. Informe de La Reunión de la OMS: La declaración de Tokio. Genebra, 1993; ORGANIZAÇÃO PAN-AMERICANA DE SAÚDE. Consenso brasileiro de atenção farmacêutica: proposta. Brasília, 2002).

${ }^{20}$ ANGONESI, D.; SEVALHO, G. Atenção farmacêutica: fundamentação conceitual e crítica para um modelo brasileiro. Ciência \& Saúde Coletiva, Rio de Janeiro, v. 15, Supl. 3, p.3603-3614, 2010.

${ }^{21}$ Nesse sentido cabe lembrar iniciativas como a do Conselho Regional de Farmácia do Estado de São Paulo em conjunto com a Organização Pan-Americana de Saúde (OPAS) em campanha pela "Farmácia como estabelecimento de saúde" e a Resolução da Diretoria Colegiada - RDC n 44 da Agência Nacional de Vigilância Sanitária (ANVISA), em 2009, que incluiu o tema na formulação de boas práticas nas farmácias. (BRASIL. Agência Nacional de Vigilância Sanitária. RDC $n^{\circ} 44$, de 17 de agosto de 2009. "Dispõe sobre Boas Práticas Farmacêuticas para o controle sanitário do funcionamento, da dispensação e da comercialização de produtos e da prestação de serviços farmacêuticos em farmácias e drogarias e dá outras providências.". Disponível em: <http://www. anvisa.gov.br/divulga/noticias/2009/pdf/180809_rdc_44.pdf>. Acesso em: 19 jan. 2014).

${ }^{22}$ FARINA S.S.; ROMANO-LIEBER N.S. Atenção Farmacêutica em Farmácias e Drogarias: existe um processo de mudança? Saúde \& Sociedade, São Paulo, v.18, n.1, p.7-18, 2009.

${ }^{23}$ TOMASSI, M.H.; RIBEIRO, E. Conhecimentos e atitudes de farmacêuticos em farmácias e drogarias do Butantã - São Paulo. Revista de Ciências Farmacêuticas Básica e Aplicada, Araraquara, v. 33, n.1, p.125-132, 2012.
} 
articular dentro de uma estrutura do setor saúde. Este, por sua vez, pressupõe ir além do dado, ou ir além do objeto de escolha imediato, o que não é comum na prática comercial corrente. Como o setor saúde se orienta pela prevenção, a assistência no ato de consumo de medicamento pode ser inclusive o direcionamento ao não consumo, fato não concebível na forma de comercialização trivial.

\section{Mercado, governo e profissão}

Aglomeração geográfica de estabelecimentos é prática comum em mercados competitivos modernos. Há razões estratégicas, tanto por fatores endógenos como exógenos. Além de permitir compartilhamentos de diferentes naturezas, a proximidade é motivada principalmente pelo melhor acesso ao consumidor. As possibilidades de interceptar suas rotas de tráfego determinam os locais estratégicos. Além disso, o empreendedor reduz seus riscos de escolha ao instalar-se ao lado de um concorrente. Ao mesmo tempo, o consumidor é atraído por locais onde há grande oferta. Nas aglomerações homogêneas, o consumidor reduz a sua expectativa de risco em relação ao preço e à disponibilidade. ${ }^{(24)}$

No Brasil, a livre concorrência entre os estabelecimentos farmacêuticos é entendida como o maior e mais eficaz instrumento de proteção e defesa do consumidor. ${ }^{(25)}$ Alguns, entretanto, criticam o crescimento e a concentração no número de estabelecimentos. Entre as alegações, vem sendo lembrado que há estímulo à automedicação e que o problema poderia ser equacionado a partir de uma lei de zoneamento. ${ }^{(26)}$ Todavia, na economia de mercado, a oferta cresce em função da demanda reprimida, baixando os preços. A relação desta com a automedicação poderia também ser entendida em sentido inverso. Pesquisas mostram que o consumidor obriga-se à automedicação, dada a precariedade do sistema de serviço de saúde. ${ }^{(27)}$ Nesses termos, o zoneamento, por ele mesmo, pouco modificaria esse quadro apontado.

Em benefício da dispersão das farmácias, investigações também vêm mostrando que as proximidades para acesso, disponibilidades de produtos, horário

\footnotetext{
${ }^{24}$ Ver Krider e Putler e Dokmeci e Ozus para a constatação desses mesmos fatores no arranjo geográfico de farmácias em Istambul. Estudo de Picone et al sugere ainda que, quanto menor a diferenciação nos produtos comercializados, mais a aglomeração força a redução dos preços. (KRIDER, R.E.; PUTLER, D.S. Which Birds of a Feather Flock Together? Clustering and Avoidance Patterns of Similar Retail Outlets. Geographical Analysis, Columbus, v. 45, n. 2, p. 123-149, 2013; DOKMECI, V.; OZUS, E. Spatial analysis of urban pharmacies in Istambul. European Planning Studies, v. 12, n. 4, p. 585-594, 2004).

${ }^{25}$ ANVISA - AGÊNCIA NACIONAL DE VIGILÂNCIA SANITÁRIA. O que devemos saber sobre medicamentos. Brasília, DF, 2010.

${ }^{26}$ XAVIER, J. Por que tanta farmácia? Radis, Rio de Janeiro, v. 29, p. 12-14, 2005. Disponível em: $<\mathrm{http}: / /$ www6.ensp.fiocruz.br/radis/revista-radis/29/reportagens/por-que-tanta-farmacia>. Acesso em: 19 set 2013.

${ }^{27}$ NAVES, J.O.S.; CASTRO, L.L.C.; CARVALHO, C.M.S., MERCHAN-HAMANN, E. Automedicação: uma abordagem qualitativa de suas motivações. Ciência \& Saúde Coletiva, Rio de Janeiro, v.15, Supl. 3, p.1751-1762, 2010.
} 
de funcionamento e outras conveniências são particularmente importantes em comunidades pobres ${ }^{(28)}$ ou não urbanas. ${ }^{(29)}$ Todavia, preço ainda importa. Populações pobres comprometem renda de forma significativa na compra de medicamentos. ${ }^{(30)}$ Enquanto, nos países desenvolvidos, as políticas públicas de fornecimento direto desse insumo vêm empenhando parte relevante do orçamento da saúde, motivando a revisão das restrições. A experiência em dois países (Islândia e Noruega) mostrou que esse processo levou a imediata concentração na atividade, maior número de estabelecimentos, principalmente nos locais já atendidos, ampliação de horários de funcionamento, redução de preços para os consumidores e nenhum benefício ao Estado, obrigado a manter os mesmos dispêndios em subsídio. ${ }^{(31)}$ Entre liberar e manter restrições, as experiências internacionais resultam nas mais diversas. A Suécia continua sob intensa restrição, mas o Estado ainda detém o monopólio sobre as farmácias comunitárias. ${ }^{(32)}$ Enquanto nos Estados Unidos a comercialização se dá preferencialmente por redes, na França esse recurso continua proibido. ${ }^{\left({ }^{33}\right)}$ AAustrália mantém restrições de zoneamento, ${ }^{(34)}$ mas no Canadá se dá o oposto, embora em ambos haja grandes variações na concentração demográfica.

Produtos farmacêuticos denotam perigos à saúde. Por um lado há o perigo decorrente da sua constituição intrínseca e do seu emprego, exigindo perícia. Por outro, há o perigo decorrente da sua falta, exigindo a livre iniciativa. Os estabelecimentos farmacêuticos impõem risco à população em relação a esses perigos e as soluções de mercado são pouco adequadas nesses casos. Mercados têm lógica própria, tendendo a externalizar riscos e transferir encargos. ${ }^{(35)}$ Para cobrir essa lacuna, o Estado depende de outros meios de controle, como a

${ }^{28}$ GOEL, P.; ROSS-DEGNAN, D.; BERMAN, P.; SOUMERAI, S. Retail pharmacies in developing countries: a behaviour and intervention framework. Social Science and Medicine, Oxford, v. 42, $\mathrm{n}$. 8, p.1155-1161, 1996.

${ }^{29}$ WATTS, P.R.; DINGER, M.K.; BALDWIN K.A.; SISK, R.J.; BROCKSCHMIDT, B.A.; MCCUBBIN, J.E. Accessibility and perceived value of health services in five Western Illinois Rural Communities. Journal of Community Health, Amsterdam. 24, n. 2, p. 147-157, 1999.

${ }^{30}$ SMITH, F. Pharmacy in developing countries. In: TAYLOR, K.M.G.; HARDING, G. (Ed.). Pharmacy practice. London: Taylor \& Francis Press, 2001. p. 71-89.

${ }^{31}$ ANELL, A. Deregulating the pharmacy market: the case of Iceland and Norway. Health Policy, Limerick, v. 75, p. 9-17, 2005.

${ }^{32}$ SWEDEN. Ministry of Health and Social Affairs Pharmacy market reform bill submitted to the Riksdag. Disponível em: http://www.government.se/sb/d/11687/a/121144. Acesso em: 19 set. 2013.

${ }^{33}$ MOSSIALOS, E.; MRAZEK, M. The Regulation of Pharmacies in Six Countries - LSE Health \& Social Care and the European Observatory on Health Care Systems. Office of Fair Trading, 2003.

${ }^{34}$ AUSTRALIAN COMMUNITY PHARMACY AUTHORITY - Pharmacy Location Rules - Applicant's Handbook. Disponível em: http://www.health.gov.au/internet/main/publishing.nsf/Content/ pharmacy-acpa-handbook-contents/\$FILE/Handbook\%20-\%20March\%202009\%20V2.pdf. Acesso em: 15 set 2013.

${ }^{35}$ Por exemplo, por meio de seguros. Solow entende que o mercado não é capaz de representar adequadamente os interesses do futuro distante. (SOLOW, R.M. Sustainability: an economist's perspective. In: DORFMAN R.; DORFMAN N. (Ed.). Economics of the Environment: Selected Readings. New York: W.W. Norton \& Company; 1993. pp. 179-87). 
relação profissional. ${ }^{(36)}$ Relações profissionais têm sido criticadas, como formas de captura do Estado para benefício de uma classe social, em semelhança às guildas. ${ }^{(37)}$ Mas o profissionalismo vem sendo cada vez mais necessário como conhecimento especializado para as situações de risco. Os procedimentos de modernização teriam excluído as certezas tradicionais e a introdução de novas certezas permitiria a organização da vida. Todavia, profissões não podem se manter estáticas, sob o risco de perda do seu papel social. ${ }^{(38)}$

\section{Considerações finais}

A organização social é um processo em contínua transformação. ${ }^{(39)}$ A maior cobertura tem elevado os custos em saúde e experiências do passado mostram trajetórias que não podem ser descartadas. ${ }^{(40)}$ Restrições de zoneamento nos dias de hoje podem ser entendidas até como irrelevantes em decorrência do comércio eletrônico, por exemplo. Com o uso assistido de algoritmos, também as restrições de exclusividade de diagnóstico por um médico ou da dispensação de medicamentos por um farmacêutico deixam de fazer sentido em locais mais carentes, foco do zoneamento. ${ }^{(41)}$ A prestação de serviço especializado, como atenção farmacêutica, pode vir a ser diferencial competitivo nesse processo, mas depende ainda de uma demanda clara, como é próprio em economias de mercado. De maneira geral, a prática nas farmácias ainda é pagar-se por um produto e não por um serviço. ${ }^{(42)}$

\footnotetext{
${ }^{36}$ DINGWALL, R. Professions and social order in a global society. International Review of Sociology, v. 9, n. 1, p. 131-140, 1999 e EVETTS, J. The sociological analysis of professionalism. International Sociology, Cardiff, v. 18, n. 2, p. 395-415, 2003.

${ }^{37}$ EVETTS, J. op. cit.

${ }^{38}$ DINGWALL, R. op. cit.

${ }^{39}$ Quer se entenda a partir da sua inerência constitutiva, ou autopoiesis, conforme Luhmann, quer se entenda na sua estruturação, ou morfogênese, conforme Buckley. (LUHMANN, N. Introdução à teoria dos sistemas. Petrópolis: Vozes, 2009. p. 121-122; BUCKLEY W. (1967). A sociologia e a moderna teoria de sistemas. São Paulo; Cutrix, 1976. p. 92).

${ }^{40}$ Assim como no passado o sistema de guildas colapsou pela insensibilidade em relação aos interesses públicos, hoje a classe médica se obriga a repensar privilégios de profissão em decorrência dos custos processo. No Reino Unido, enquanto a tradição prescritora do farmacêutico deu origem ao atual "prático geral" (TOWNSEND, E. op. cit.; COWEN, D.L.; HELFAND, W.H. op. cit., p. 108), as desregulamentações surgidas a partir de 1986 vêm permitindo a prescrição pelo próprio farmacêutico, em semelhança ao boticário medieval inglês (ANDERSON, S. op. cit.). No Brasil, vigora a Resolução $n^{\circ} 586 / 13$ do Conselho Federal de Farmácia, que regulamenta a prescrição de medicamentos por farmacêuticos. (BRASIL. Conselho Federal de Farmácia. Resolução $n^{\circ}$ 586, de 29 de agosto de 2013. Disponível em: <http://www.cff.org.br/userfiles/file/ noticias/Resolu\%C3\%A7\%C3\%A30586_13.pdf>. Acesso em: 19 jan. 2014.

${ }^{41}$ Clínicas populares nos EUA oferecem, em horários convenientes, serviços rápidos e baratos de diagnósticos e indicação terapêutica por meio de algoritmos conduzidos usualmente por enfermeiros sob supervisão médica à distância. (POLLACK, C.E.; ARMSTRONG, K. The geographic accessibility of retail clinics for underserved populations. Archives of Internal Medicine, Chicago, v. 169, n. 10, p. 945-949, 2009).

${ }^{42}$ ANDERSON, S. op. cit.
} 
No Brasil, em particular, a mídia já expõe a insensatez das restrições profissionais nas intervenções mais prosaicas ligadas à medicina e ao direito. A preferência pela exclusão absoluta dos riscos via viés profissional desampara e onera a grande maioria dos necessitados carentes de atendimento. ${ }^{(43)}$ Esse é o risco do controle. Em compensação, novas tecnologias, novas práticas comerciais, novos perfis de produção e consumo são introduzidos no âmbito da população a ser protegida. Excluir as incertezas associadas, mesmo as de conflitos de interesse médico/farmacêutico, implica também em se excluírem oportunidades. Na ação do Estado, portanto, essas incertezas precisam ser identificadas, qualificadas e contingenciadas em relação aos riscos potenciais. Em outras palavras, o caminho fácil da exclusão das incertezas pelas regras deve, cada vez mais, dar lugar ao controle dos riscos que possam estar associados a estas incertezas inevitáveis.

\section{Referências}

ABEL, R. L. A socialist approach to risk. Maryland Law Review, Maryland, v.41, p. 695-754, 1981-1982.

ANDERSON, S. The historical context of pharmacy. In: TAYLOR, K. MG; HARDING, G. (Ed.). Pharmacy Practice. London: Taylor \& Francis Press, 2001, p. 3-27.

ANELL, A. Deregulating the pharmacy market: the case of Iceland and Norway. Health Policy, Limerick, v. 75, p. 9-17, 2005.

ANGONESI, D.; SEVALHO, G. Atenção farmacêutica: fundamentação conceitual e crítica para um modelo brasileiro. Ciência \& Saúde Coletiva, Rio de Janeiro, v. 15, Supl. 3, p.3603-3614, 2010.

ANVISA - AGÊNCIA NACIONAL DE VIGILÂNCIA SANITÁRIA. O que devemos saber sobre medicamentos. Brasília, DF, 2010.

AUSTRALIAN COMMUNITY PHARMACY AUTHORITY - Pharmacy Location Rules - Applicant's Handbook. Disponível em: http://www.health.gov.au/ internet/main/publishing.nsf/Content/pharmacy-acpa-handbook-contents/\$FILE/ Handbook\%20-\%20March\%202009\%20V2.pdf. Acesso em: 15 set 2013.

BUCKLEY W. (1967). A sociologia e a moderna teoria de sistemas. São Paulo; Cutrix, 1976.

COWEN, D.L. Pharmacists and physicians: an uneasy relationship. Pharmacy in History, Madison, v. 34, n. 1, p. 3-16, 1992.

Pharmacy and freedom. Pharmacy in History, Madison v. 26, n. 2, p. 70-82, 1984.

\footnotetext{
${ }^{43}$ SCHWARTSMAN, H. Novos tempos. Folha de S. Paulo, São Paulo, 28 set 2013. Disponível em: <http://www1.folha.uol.com.br/colunas/helioschwartsman/2013/09/1348637-novos-tempos.shtml>. Acesso em: 28 set 2013.
} 
COWEN, D.L. A store mixt, various, universal. The Journal of the Rutgers University Libraries, New Brunswick, v. 25, n. 1, p. 1-9, dez. 1961.

; HELFAND, W.H. Pharmacy: an illustrated history. New York: Abrams Inc; 1990.

DINGWALL, R. Professions and social order in a global society. International Review of Sociology, v. 9, n. 1, p. 131-140, 1999.

DOKMECI, V.; OZUS, E. Spatial analysis of urban pharmacies in Istambul. European Planning Studies, v. 12, n. 4, p. 585-594, 2004.

DÜZBAKAR, Ö.; ERCAN, Ö. Notes on the Attar-Poets in ottoman history: reflections from the Shari'a court records of bursa and poets' biographies. Journal of the International Society for the History of Islamic Medicine, Istambul, v.5, p.19-24, 2006.

EPSTEIN, S.R. Craft guilds, apprenticeship, and technological change in preindustrial Europe. Journal of Economic History, Cambridge, v. 58, p. 684-713, 1998.

EVETTS, J. The sociological analysis of professionalism. International Sociology, Cardiff, v. 18, n. 2, p. 395-415, 2003.

FARINA S.S.; ROMANO-LIEBER N.S. Atenção Farmacêutica em Farmácias e Drogarias: existe um processo de mudança? Saúde \& Sociedade, São Paulo, v.18, n.1, p.7-18, 2009.

GOEL, P.; ROSS-DEGNAN, D.; BERMAN, P.; SOUMERAI, S. Retail pharmacies in developing countries: a behaviour and intervention framework. Social Science and Medicine, Oxford, v. 42, n. 8, p.1155-1161, 1996.

HEPLER, C.D.; STRAND, L.M. Oportunidades y responsabilidades en la Atención Farmacéutica. American Journal of Hospital Pharmacy, Bethesda, v.47, p. 533-534, 1990.

KRIDER, R.E.; PUTLER, D.S. Which Birds of a Feather Flock Together? Clustering and Avoidance Patterns of Similar Retail Outlets. Geographical Analysis, Columbus, v. 45, n. 2, p. 123-149, 2013.

LIEBER R.R.; ROMANO-LIEBER N.S. Risco e precaução no desastre tecnológico. Cadernos de Saúde Coletiva, Rio de Janeiro, v.13, n.1, p.67-84, 2005.

LUHMANN, N. Introdução à teoria dos sistemas. Nasser. Petrópolis: Vozes, 2009.

MOSSIALOS, E.; MRAZEK, M. The Regulation of Pharmacies in Six Countries - LSE Health \& Social Care and the European Observatory on Health Care Systems. Office of Fair Trading, 2003. 48p.

NAVES, J.O.S.; CASTRO, L.L.C.; CARVALHO, C.M.S., MERCHAN-HAMANN, E. Automedicação: uma abordagem qualitativa de suas motivações. Ciência \& Saúde Coletiva, Rio de Janeiro, v.15, Supl. 3, p.1751-1762, 2010. 
ORGANIZAÇÃO PAN-AMERICANA DE SAÚDE. Consenso brasileiro de atenção farmacêutica: proposta. Brasília, 2002.

ORGANIZACIÓN MUNDIAL DE LA SALUD. El papel del farmacéutico en el sistema de atención de salud. Informe de La Reunión de la OMS: La declaración de Tokio. Genebra, 1993.

POLLACK, C.E.; ARMSTRONG, K. The geographic accessibility of retail clinics for underserved populations. Archives of Internal Medicine, Chicago, v. 169, n. 10, p. 945-949, 2009.

SCHEPERS, R. Pharmacists and medical doctors in nineteenth century Belgium. Sociology of Health \& IIIness, Leeds, v. 10, n. 1, p. 68-90, 1988.

SCHWARTSMAN, H. Novos tempos. Folha de S. Paulo, São Paulo, 28 set 2013. Disponível em: <http://www1.folha.uol.com.br/colunas/ helioschwartsman/2013/09/1348637-novos-tempos.shtml>. Acesso em: 28 set 2013.

SMITH, F. Pharmacy in developing countries. In: TAYLOR, K.M.G.; HARDING, G. (Ed.). Pharmacy Practice. London: Taylor \& Francis Press, 2001. p. 71-89.

SOLOW, R.M. Sustainability: an economist's perspective. In: DORFMAN R.; DORFMAN N. (Ed.). Economics of the Environment: Selected Readings. New York: W.W. Norton \& Company; 1993. pp. 179-87. Sox (2007).

SWEDEN. Ministry of Health and Social Affairs Pharmacy market reform bill submitted to the Riksdag. Disponível em: http://www.government.se/ sb/d/11687/a/121144. Acesso em 19 set 2013.

TOMASSI, M.H.; RIBEIRO, E. Conhecimentos e atitudes de farmacêuticos em farmácias e drogarias do Butantã - São Paulo. Revista de Ciências Farmacêuticas Básica e Aplicada, Araraquara, v. 33, n.1, p.125-132, 2012.

TOWNSEND, E. Future trends in general practice. The Journal of the College of General Practitioners, London, v. 5, n. 4, p. 501-24, 1962.

VIEIRA, F.S. Possibilidades de contribuição do farmacêutico para a promoção da saúde. Ciência \& Saúde Coletiva, Rio de Janeiro, v.12, n.1, p.213-220, 2007.

WATTS, P.R.; DINGER, M.K.; BALDWIN K.A.; SISK, R.J.; BROCKSCHMIDT, B.A.; MCCUBBIN, J.E. Accessibility and perceived value of health services in five Western Illinois Rural Communities. Journal of Community Health, Amsterdam. 24, n. 2, p. 147-157, 1999.

XAVIER, J. Por que tanta farmácia? Radis, Rio de Janeiro, v. 29, p. 12-14, 2005. Disponível em: <http://www6.ensp.fiocruz.br/radis/revista-radis/29/reportagens/ por-que-tanta-farmacia>. Acesso em: 19 set 2013. 